\title{
Relative Filling Time Delay Based on CT Perfusion Source Imaging: A Simple Method to Predict Outcome in Acute Ischemic Stroke
}

\author{
W. Cao, B.C.V. Campbell, Q. Dong, S.M. Davis, and B. Yan
}

\begin{abstract}
BACKGROUND AND PURPOSE: Collateral vessel status is strongly associated with clinical outcome in ischemic stroke but can be challenging to assess. The aim of this study was to develop a tomography perfusion source imaging-based assessment of collateral vessel status.

MATERIALS AND METHODS: Consecutive patients with ischemic stroke who received intravenous thrombolysis or intra-arterial reperfusion therapy after CTP were retrospectively analyzed. In those with middle cerebral artery or internal carotid artery occlusion, CT perfusion source imaging was used to identify the relative filling time delay between the normal MCA Sylvian branches and those in the affected hemisphere. Receiver operating characteristic analysis and logistic regression were used to assess the association of the relative filling time delay with the 24-hour Alberta Stroke Program Early CT Score based on noncontrast CT and the 90-day modified Rankin Scale score.

RESULTS: There were 217 patients treated in 2009-2011 who had CTP data, of whom 60 had MCA or ICA occlusion and 55 had 90 -day $\mathrm{mRS}$ data. The intraclass correlation coefficient for relative filling time delay was 0.95 . Relative filling time delay was correlated with 24-hour ASPECTS (Spearman $\rho=-0.674 ; P<.001)$ and 90 -day mRS score $(\rho=0.516, P<.01)$. Increased relative filling time delay was associated with poor radiologic outcome (ASPECTS, $0-7$ ) (area under the curve $=0.79, P<.001$ ) and poor functional outcome (mRS score, 3-6) (area under the curve $=0.77, P=.001$ ). In multivariate logistic regression, the association of longer relative filling time delay with poor outcome remained significant, independent of age, sex, and baseline National Institutes of Health Stroke Scale score.
\end{abstract}

CONCLUSIONS: Relative filling time delay is a useful independent predictor of clinical outcome after ischemic stroke.

ABBREVIATIONS: $C T P-S I=C T$ perfusion source imaging; $r F T D=$ relative filling time delay

eptomeningeal collateral flow has an important role in main-

taining blood flow to brain regions distal to an arterial occlusion. ${ }^{1-5}$ Imaging assessment of leptomeningeal collaterals in humans does not depict the small interarteriolar connections directly but, instead, relies on an indirect assessment of the extent and rate of backfilling of pial arteries receiving blood flow through these collateral vessels. ${ }^{1,4,6,7}$ Many leptomeningeal collateral flow studies, most of which were CT angiography studies, used various grading methods to assess the vessel filling status in the Sylvian fissure by observers. ${ }^{3,8-11}$ Traditional assessment of leptomenin-

Received October 15, 2013; accepted after revision February 6, 2014.

From the Department of Medicine and Neurology, Melbourne Brain Centre at the Royal Melbourne Hospital (W.C., B.C.V.C., S.M.D., B.Y.), University of Melbourne, Parkville, Victoria, Australia; and Department of Neurology (W.C., Q.D.), Huashan Hospital, Shanghai, China.

Please address correspondence to Bernard Yan, MD, Royal Melbourne Hospital, Parkville, Victoria 3050, Australia; e-mail: Bernard.yan@mh.org.au

http://dx.doi.org/10.3174/ajnr.A3931 geal collateral flow by using static CTA images lacks temporal resolution. Although newer scanners offer whole-brain perfusion acquisitions that can be reconstructed to provide dynamic CTA by using advanced software, ${ }^{12}$ this technology is not yet widely available.

CT perfusion expands the role of CT in the evaluation of acute stroke by providing physiologic insights into cerebral hemodynamics and, in so doing, complements the strength of CTA by determining the consequences of vessel occlusions and stenosis. ${ }^{9,12,13}$ Although 1 study used CTP source data to confirm that contrast opacification was indeed retrograde collateral flow, no prior studies have graded collateral status by rating CTP source imaging (CTP-SI). We investigated a simple, time-resolved scale of collateral-derived contrast opacification in the Sylvian fissure as a predictor of radiologic and functional outcome. We hypothesized that delayed filling of the middle cerebral artery in the Sylvian fissure due to poor collateral flow would be associated with worse radiologic and functional outcome after ischemic stroke. 


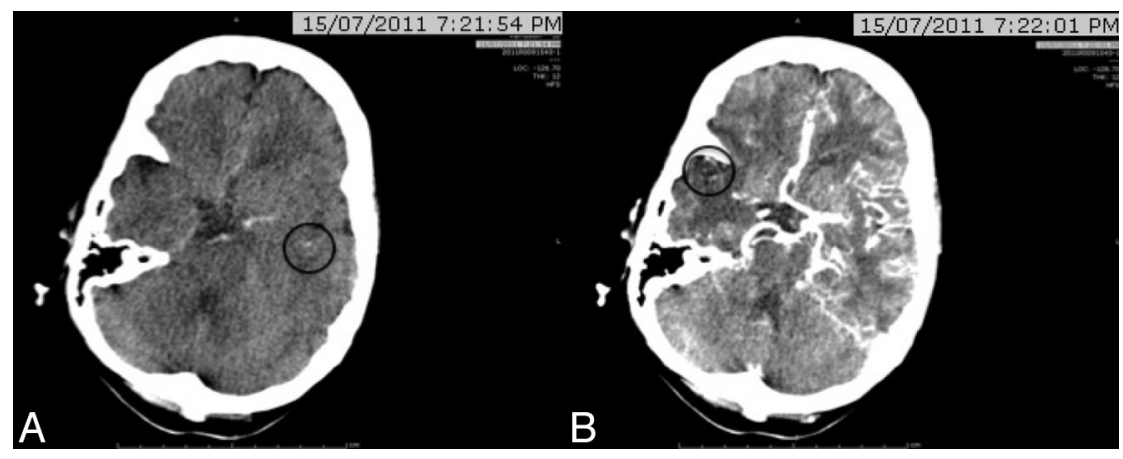

FIG 1. A 67-year-old woman with left hemiparesis and dysarthria who presented to the hospital 2 hours after onset. CTA identified a right intracranial ICA occlusion. CTP-SI showed that the contrast (black circles) was first detected at 20 seconds in the left Sylvian fissure $(A)$ and at 27 seconds in the affected right hemisphere $(B)$. The relative filling time delay was, therefore, 7 seconds.

between observers (with a tolerance of \pm 1 -second difference) was calculated. Cases with 1-second difference between the 2 raters were averaged, and those with $\geq 2$-second difference were reviewed by an interventional neuroradiologist (B.Y.) to determine consensus. The Alberta Stroke Program Early CT Score of 24hour NCCT was independently assessed by the neurointerventionalist and stroke neurologist blinded to all other information before consensus was reached.

\section{Statistical Analysis}

Statistical analyses were performed by using the Statistical Package for the Social

\section{MATERIALS AND METHODS Patients}

We identified patients with acute ischemic stroke presenting to our institution between January 2009 and December 2011 within 4.5 hours of stroke-symptom onset who underwent multimodal CT imaging before reperfusion therapy from our prospectively recorded stroke data base. The subgroup with MCA or internal carotid artery occlusion was selected for this analysis. Patients with MCA occlusion of the M1 or both M2 segments proximal to the Sylvian fissure were included. Baseline National Institutes of Health Stroke Scale scores and 90-day modified Rankin Scale scores were obtained from the data base. The study was approved by the Melbourne Health Human Research Ethics Committee.

\section{Imaging}

Noncontrast CT, CT perfusion, and CT angiography were performed before therapy. Two separate CTP slabs, each 24-mm thick, were acquired consecutively (16-section Somatom CT; Siemens, Erlangen, Germany; 80 kV(peak), $209 \mathrm{mAs}$ ) and positioned to maximize supratentorial coverage. Iodinated contrast $(40-\mathrm{mL}$ iohexol 350 mg I/mL, Omnipaque 350; GE Healthcare, Milwaukee, Wisconsin) was injected at $8 \mathrm{~mL} / \mathrm{s}$, and 40 images were acquired every second (total acquisition time, 44 seconds). Each slab was formatted as two 12-mm sections. Follow-up NCCT was performed at a minimum of 24 hours after onset.

\section{Imaging Analysis}

NCCT, CTP, and CTA were reviewed by using standard PACS software, allowing reviewers to freely window and view the images. CTA images were reviewed by 1 stroke neurologist, to identify patients with complete (Thrombolysis in Myocardial Infarction, 0 ) occlusion of the MCA or ICA. In patients with MCA or ICA occlusion, review of cervical CTA did not detect any cases with moderate-to-severe stenosis $(>50 \%)$, which might have confounded relative filling time delay (rFTD) interpretation. Unprocessed CTP-SI DICOM images were used to identify the relative filling time delay between the normal MCA Sylvian branches and those in the affected hemisphere. It was calculated as the time difference between the first appearance of contrast in each Sylvian fissure (Fig 1). rFTD was then independently assessed by a neurointerventionalist (W.C.) and a stroke neurologist (B.C.V.C.), blinded to clinical outcomes. The agreement in rFTD assessment
Sciences, Version 19 (IBM, Armonk, New York), and STATA, Version 12 (StataCorp, College Station, Texas). $P<.05$ was considered to indicate statistical significance. Interobserver agreement (with tolerance of \pm 1 -second difference) for the assessment of rFTD between the 2 observers was tested by using Cohen $\kappa$ and intraclass correlation. Patients were dichotomized by using the 90-day mRS score into good ( $\mathrm{mRS}$ score, $0-2$ ) versus poor ( $\mathrm{mRS}$ score, 3-6) outcome, and mortality (mRS score, 6) was analyzed separately. ${ }^{14}$ The 24-hour ASPECTS was dichotomized into good (ASPECTS, 8-10) versus poor (ASPECTS, 0-7) radiologic outcomes. ${ }^{15,16}$ Differences in patient characteristics between outcomes were tested by the Fisher exact test for categoric and the Mann-Whitney $U$ test for continuous values. Comparisons of rFTD and baseline NIHSS scores between groups were performed by using the Mann-Whitney $U$ test. The Spearman nonparametric rank correlation was performed to assess the correlation between rFTD and baseline NIHSS scores, 90-day mRS scores, and 24hour ASPECTS. Multivariate binary logistic regression (including variables with $P$ values $<.05)$ was used to assess the association of rFTD with 24-hour ASPECTS and 90-day mRS scores. Receiver operating characteristic analysis was performed to determine the optimal threshold. Positive predictive value, sensitivity, and specificity at the optimal threshold were calculated.

\section{RESULTS}

Between January 2009 to December 2011, two hundred seventeen patients with acute ischemic stroke presented to our institution within 4.5 hours of stroke-symptom onset, were imaged with CTP, and then received intravenous tPA or intra-arterial therapy. Of those, 60 patients with acute occlusion identified in the ICA or MCA were included in this study. The mean patient age was $70 \pm$ 10 years with 27/60 (45\%) being women. Hypertension was found in $58.3 \%$ (35/60); diabetes mellitus, in $28.3 \%$ (17/60); hyperlipidemia, in 55\% (33/60); atrial fibrillation, in 38.3\% (23/60); and current or past smoking, in $18.3 \%(11 / 60)$ of the patients. The baseline median pretreatment NIHSS score was 16 (interquartile range, 3-27). Mean onset-to-therapy time was $141 \pm 48$ minutes. There were 41 patients $(68.3 \%)$ treated with intravenous recombinant tissue plasminogen activator, and 19 patients $(31.7 \%)$ received intra-arterial therapy, including 5 (8.3\%) with rtPA/urokinase and $14(23.3 \%)$ with mechanical thrombectomy. The site of 


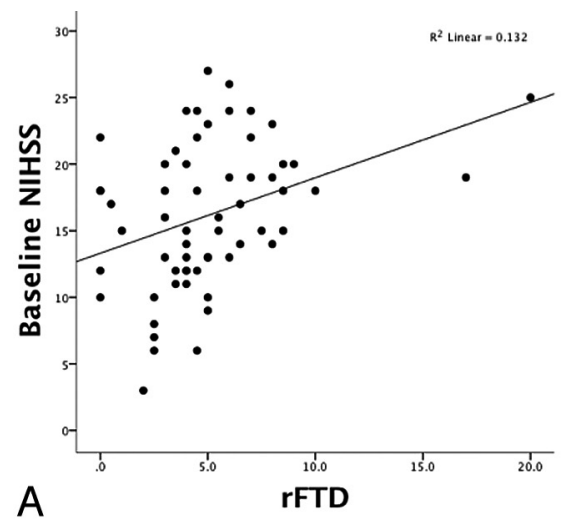

Spearman's rho $=0.407, p=0.001$

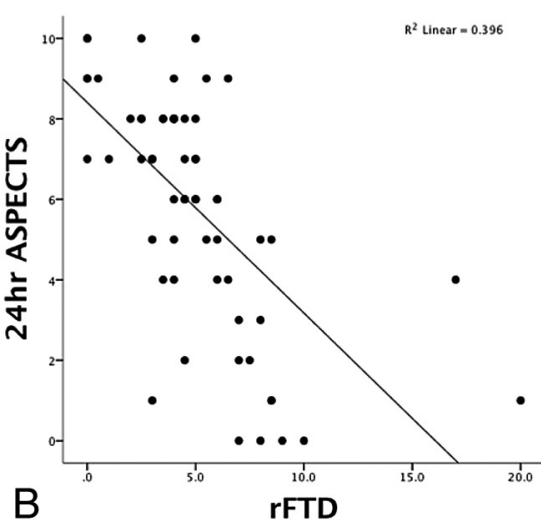

Spearman's rho $=-0.674, p<0.001$

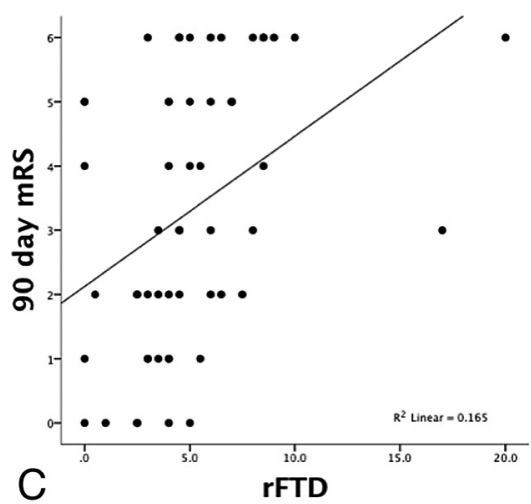

Spearman's rho $=0.516, p<0.001$

FIG 2. Correlation between rFTD and baseline NIHSS scores, 24-hour ASPECTS, and 90-day mRS scores.

Table 1: Comparison of patient characteristics in those with good-versus-poor outcomes

\begin{tabular}{|c|c|c|c|c|c|c|c|c|c|}
\hline & $\begin{array}{c}\mathrm{mRS}(0-2) \\
(n=23)\end{array}$ & $\begin{array}{c}\mathrm{mRS}(3-6) \\
(n=32)\end{array}$ & $P$ Value & $\begin{array}{c}\text { Alive } \\
(n=43)\end{array}$ & Dead $(n=12)$ & $P$ Value & $\begin{array}{l}\text { ASPECTS } \\
(>7)(n=21)\end{array}$ & $\begin{array}{c}\text { ASPECTS } \\
(\leq 7)(n=39)\end{array}$ & $P$ Value \\
\hline Age (median) (yr) & $69(41-85)$ & $73.5(54-88)$ & .014 & $69(41-85)$ & $79(66-88)$ & .005 & $72(52-83)$ & $73(41-88)$ & .877 \\
\hline Female (\%) & 26 & 56.2 & .026 & 37.2 & 66.6 & .101 & 33.3 & 51.2 & .277 \\
\hline Hypertension (\%) & 52.1 & 62.5 & .444 & 60.4 & 50 & .516 & 56.4 & 61.9 & .786 \\
\hline Diabetes mellitus (\%) & 26 & 28.1 & .867 & 23.2 & 41.6 & .205 & 33.3 & 25.6 & .560 \\
\hline Hyperlipidemia (\%) & 60.8 & 46.8 & .305 & 48.8 & 66.6 & .274 & 61.9 & 51.2 & .587 \\
\hline Current or past smoker (\%) & 26 & 9.3 & .143 & 20.9 & 0 & .181 & 14.2 & 20.5 & .731 \\
\hline Atrial fibrillation (\%) & 34.3 & 34.7 & .975 & 32.5 & 41.7 & .733 & 42.8 & 35.8 & .781 \\
\hline Baseline NIHSS (median) & $13(6-20)$ & $19(6-27)$ & .001 & $15(6-27)$ & $21(15-26)$ & .001 & $12(3-24)$ & $18(6-27)$ & .003 \\
\hline OTT (median) (min) & $120(61-255)$ & $140(75-265)$ & .366 & $140(61-265)$ & $124(77-200)$ & .683 & $137(61-250)$ & $140(76-265)$ & .364 \\
\hline IV-tPA (\%) & 60.9 & 71.9 & .561 & 67.4 & 66.7 & 1.000 & 69.2 & 66.7 & 1.000 \\
\hline IA-tPA/UK (\%) & 13 & 6.2 & .639 & 7.3 & 16.7 & .298 & 11.7 & 8.3 & 1.000 \\
\hline Clot retrieval (\%) & 26.1 & 21.9 & .717 & 25.5 & 16.7 & .709 & 23.1 & 23.8 & 1.000 \\
\hline rFTD (median) (sec) & $3.5(0-7.5)$ & $5.75(0-10)$ & .001 & $4(0-17)$ & $6.5(3-20)$ & .002 & $3.5(0-6.5)$ & $5.5(2-20)$ & .002 \\
\hline
\end{tabular}

Note:-IA indicates intra-arterial; rFTD, relative filling delay time; UK, urokinase; OTT, onset to treatment time.

arterial occlusion on CTA was in the proximal (M1) MCA in 44 $(73.3 \%)$ patients, the distal MCA (M2) in $8(13.3 \%)$ patients, and the ICA in $8(13.3 \%)$ patients. The 90 -day mRS score was available in 55 patients. There was no significant difference in baseline characteristics in these patients compared with the entire population. Good functional outcome (mRS score, $0-2$ ) was seen in 23 patients $(41.8 \%)$, and death occurred in $21.8 \%(12 / 55)$.

Exact agreement of rFTD between the 2 observers was found in 32 of 60 patients $(\kappa=0.47, P<.001)$. Tolerance of a $\pm 1-$ second difference increased agreement to 52 of 60 patients $(\kappa=$ $0.82, P<.001$ ). The intraclass correlation coefficient was 0.95 , which represents excellent agreement between raters.

The variables in 60 patients with 24-hour ASPECTS and 55 patients with 90-day mRS scores and mortality were analyzed. Longer rFTD was correlated with increased baseline NIHSS scores (Spearman $\rho=0.407, P=.001)$, lower 24-hour ASPECTS $(\rho=$ $-0.674, P<.001)$, and higher 90 -day mRS scores $(\rho=0.516, P<$ .001 , Fig 2 ). In the 2 outliers with rFTD values $>15$ seconds, one had contrast filling detected at 17 seconds and the other had no contrast filling and was conservatively scored at 20 seconds based on the time between perfusion of the opposite Sylvian fissure and the end of the perfusion acquisition. Excluding these 2 outliers, rFTD remained significantly correlated with baseline NIHSS score $(\rho=0.37, P=.004), 24$-hour ASPECTS $(\rho=-0.654, P<$ $.001)$, and 90 -day mRS score $(\rho=0.511, P<.001)$.

Patient characteristics stratified by outcome are listed in
Table 1. As expected, increased age and higher baseline NIHSS scores were significantly associated with worse functional and mortality outcomes. Female sex was associated with worse functional outcome. Higher baseline NIHSS scores were strongly associated with worse radiologic outcome $(P=.003)$. Longer median $\mathrm{rFTD}$ was significantly found in worse functional, mortality, and radiologic outcomes (Fig 3). In multivariate logistic regression, rFTD was associated with functional and radiologic outcomes after adjustment for age, sex, and baseline NIHSS scores (Table 2).

In receiver operating characteristic analysis, increased $\mathrm{rFTD}$ was associated with 24-hour ASPECTS of $\leq 7$ (area under curve $=$ 0.79; 95\% CI, 0.68-0.9; $P<.001$ ), 90-day mRS scores (3-6) (area under the curve $=0.77 ; 95 \% \mathrm{CI}, 0.65-0.9 ; P=.001)$, and 90 -day mortality (area under the curve $=0.79 ; 95 \%$ CI, 0.65-0.93; $P=$ .002). The optimal threshold determined by the Youden index was 4.25 seconds for both functional and radiologic outcomes.

The threshold of $\mathrm{rFTD}$ of $>4$ seconds predicted poor functional outcome ( $\mathrm{mRS}$ score, 3-6) with a sensitivity of $75 \%(95 \%$ CI, $56 \%-88 \%$ ), a specificity of $73 \%$ (95\% CI, $51 \%-89 \%)$, and a positive predictive value of $80 \%$ (95\% CI, 61\%-92\%); predicted mortality with a sensitivity of $91 \%$ (95\% CI, 61\%-99\%), a specificity of $55 \%$ ( $95 \%$ CI, 39\%-70\%), and a positive predictive value of 36\% (95\% CI, 19\%-56\%); and predicted poor radiologic outcome (ASPECTS, 0-7) with a sensitivity of 71\% (95\% CI, 55\%- 

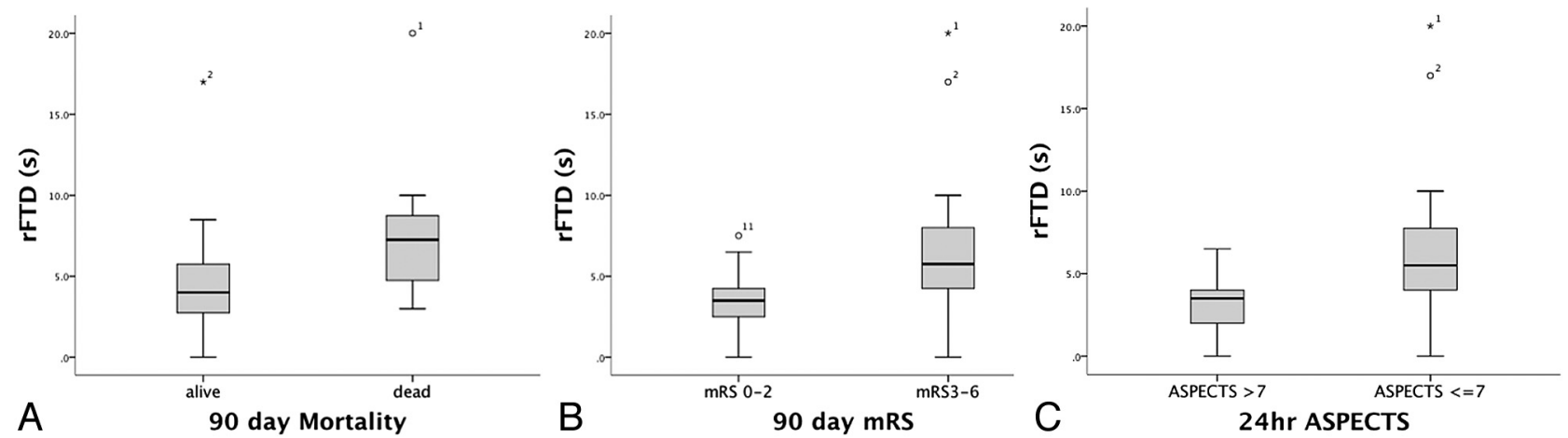

FIG 3. Longer median rFTD was significantly associated with poor functional and radiologic outcomes.

Table 2: Multivariate logistic regression for clinical and radiologic outcome

\begin{tabular}{clllc}
\hline Poor Outcomes & Predictors & $\boldsymbol{\beta}$ & \multicolumn{1}{c}{ SE } & $\boldsymbol{P}$ Value \\
\hline 90-Day mRS 3-6 & Age & 0.097 & 0.043 & .024 \\
& Sex & 0.548 & 0.816 & .501 \\
& Baseline NIHSS & 0.207 & 0.091 & .023 \\
90-Day death & rFTD & 0.354 & 0.178 & .047 \\
& Age & 0.185 & 0.074 & .01 \\
& Baseline NIHSS & 0.239 & 0.116 & .028 \\
24-Hour ASPECTS $\leq 7$ & rFTD & 0.302 & 0.158 & .039 \\
& Baseline NIHSS & 0.114 & 0.065 & .082 \\
& rFTD & 0.456 & 0.162 & .005 \\
\hline
\end{tabular}

$85 \%$ ), a specificity of $76 \%$ (95\% CI, $52 \%-91 \%)$, and a positive predictive value of $84 \%$ (95\% CI, $68 \%-94 \%$ ).

\section{DISCUSSION}

We found that a simple visual rating of collateral flow by using CTP source images is significantly associated with clinical and radiologic outcome in ischemic stroke. The relative filling time delay assessed in the Sylvian fissure represents the abundance of leptomeningeal collateral supply. Longer rFTD was associated with adverse outcomes, independent of standard prognostic variables, including age and baseline NIHSS scores. These results suggest that rFTD, especially $>4$ seconds, may be clinically useful as a marker of poor prognosis after ischemic stroke with proximal vessel occlusion.

Several studies have established the importance of collateral supply in the prediction of clinical and radiologic outcome after ischemic stroke. ${ }^{17-19}$ Collateral blood flow plays a pivotal role in the pathophysiology of cerebral ischemia but is difficult to quantify due to its complexity. In this study, we developed a method to evaluate the collateral status in patients with acute ischemic stroke with MCA or ICA occlusions by using CTP-SI. In contrast to standard CTA, which is a single "snapshot" of cerebral blood flow, CTP-SI provides a dynamic view of filling in the intracranial collateral arteries. Additionally, previous CTA studies used a variety of methods for grading leptomeningeal collateral flow, and limited data on interobserver agreement are available. ${ }^{20}$ We developed rFTD as a marker to assess the collateral circulation. The simplicity of rating the first appearance of contrast minimized observer subjectivity and translated to excellent inter-rater agreement for rFTD. The variability in the assessment of contrast-appearance time between raters was generally $<2$ seconds. A more quantitative rFTD could be obtained by using time-attenuation curve analysis with a manually positioned region of interest, though this would require dedicated software. Because $\mathrm{rFTD}$ is normalized to contralateral delay, factors such as the contrast injection and cardiac output, which affect the absolute delay to filling, are negated.

Studies of CTP in acute stroke commonly focus on 4 perfusion map-based parameters: cerebral blood volume, cerebral blood flow, mean transit time, and time-to-peak enhancement, with the aim of distinguishing salvageable tissue (penumbra) from the irreversibly injured ischemic core. The use of CTP-SI in evaluating collateral blood flow has been largely untested but has the advantage of combining anatomic information with temporal resolution to assess both the structure and function of collateral pathways. Assessing rFTD based on CTP-SI is also robust to motion in uncooperative patients. No special software is needed to perform CTP-SI assessment, so the method is feasible in most hospitals.

Recently, timing-invariant CTA was used to obtain CTA images from the CT perfusion source data that are insensitive to the timing of contrast arrival ${ }^{21}$ and, therefore, display collateral vessels that are undetectable on standard CTA. With a visual 4-point grading scale, poor retrograde collateral flow on timing-invariant CTA was associated with poor clinical outcome. ${ }^{21}$ However, the temporal information provided by CTP is a key strength, and timing-invariant CTA does not consider the potential impact of delay, which, in our data, seems highly predictive of outcome. There are more sophisticated methods to assess collateral flow by using whole-brain CTP. ${ }^{22}$ However, these require 320-slice CT, which would limit their applicability. In contrast, rFTD assessment can be performed by using any CT scanner technology and is simple and robust in clinical practice.

There are several limitations of this study. First, this is a retrospective study in a selected population of patients with ischemic stroke with ICA or MCA occlusion. Second, the rFTD method is limited to large-vessel-occlusion stroke, and the utility and value of $r F T D$ requires validation in an independent cohort. rFTD focuses on the Sylvian fissure and does not account for more peripherally located collateral flow. Finally, recanalization following treatment was not analyzed in this study because repeat vascular imaging was not routinely performed in clinical practice. Variable recanalization and the mix of IV-tPA and endovascular therapy may have potentially affected the outcome independent of collateral status. The recent neutral endovascular trials such as the Interventional Management of Stroke III trial ${ }^{23}$ and the Mechanical 
Retrieval and Recanalization of Stroke Clots Using Embolectomy (MR-RESCUE) trial ${ }^{24}$ suggest that rates of recanalization between IV and intra-arterial-treated patients during the period of our study were not likely to be very different. However, any effect of recanalization heterogeneity would have reduced the strength of association observed between rFTD and the clinical and radiologic outcomes, and our results would, therefore, be a conservative estimate of the prognostic value of rFTD.

\section{CONCLUSIONS}

rFTD obtained by CTP-SI is a simple method to quantify cerebral collateral circulation and help overcome the lack of temporal resolution of CTA for collateral assessment. Patients with longer rFTD are at increased risk of developing adverse radiologic and functional outcomes in the future. However, further studies are needed to validate our findings in an independent cohort of patients.

Disclosures: Stephen M. Davis—UNRELATED: Consultancy: Boehringer Ingelheim, EVER Pharma, Covidien, Payment for Lectures (including service on Speakers Bureaus): Boehringer Ingelheim, EVER Pharma, Covidien.

\section{REFERENCES}

1. Christoforidis GA, Mohammad Y, Kehagias D, et al. Angiographic assessment of pial collaterals as a prognostic indicator following intra-arterial thrombolysis for acute ischemic stroke. AJNR Am J Neuroradiol 2005;26:1789-97

2. Maas MB, Lev MH, Ay H, et al. Collateral vessels on CT angiography predict outcome in acute ischemic stroke. Stroke 2009;40:3001-05

3. Miteff F, Levi CR, Bateman GA, et al. The independent predictive utility of computed tomography angiographic collateral status in acute ischaemic stroke. Brain 2009;132:2231-38

4. Menon BK, Smith EE, Modi J, et al. Regional leptomeningeal score on CT angiography predicts clinical and imaging outcomes in patients with acute anterior circulation occlusions. AJNR Am J Neuroradiol 2011;32:1640-45

5. Bang OY, Saver JL, Kim SJ, et al. Collateral flow predicts response to endovascular therapy for acute ischemic stroke. Stroke 2011;42: 693-99

6. Kim JJ, Fischbein NJ, Lu Y, et al. Regional angiographic grading system for collateral flow: correlation with cerebral infarction in patients with middle cerebral artery occlusion. Stroke 2004;35: $1340-44$

7. Tan JC, Dillon WP, Liu S, et al. Systematic comparison of perfusion-CT and CT-angiography in acute stroke patients. Ann Neurol 2007;61:533-43

8. Knauth M, von Kummer R, Jansen O, et al. Potential of CT angiography in acute ischemic stroke. AJNR Am J Neuroradiol 1997; $18: 1001-10$
9. Wildermuth S, Knauth M, Brandt T, et al. Role of CT angiography in patient selection for thrombolytic therapy in acute hemispheric stroke. Stroke 1998;29:935-38

10. Rosenthal ES, Schwamm LH, Roccatagliata L, et al. Role of recanalization in acute stroke outcome: rationale for a CT angiogram-based "benefit of recanalization" model. AJNR Am J Neuroradiol 2008; 29:1471-75

11. Lima FO, Furie KL, Silva GS, et al. The pattern of leptomeningeal collaterals on CT angiography is a strong predictor of long-term functional outcome in stroke patients with large vessel intracranial occlusion. Stroke 2010;41:2316-22

12. Frölich AM, Schrader D, Klotz E, et al. 4D CT angiography more closely defines intracranial thrombus burden than single-phase CT angiography. AJNR Am J Neuroradiol 2013;34:1908-13

13. Lev MH, Farkas J, Rodriguez VR, et al. CT angiography in the rapid triage of patients with hyperacute stroke to intraarterial thrombolysis: accuracy in the detection of large vessel thrombus. J Comput Assist Tomogr 2001;25:520-28

14. Anderson CS, Heeley E, Huang Y, et al. Rapid blood-pressure lowering in patients with acute intracerebral hemorrhage. $N$ Engl J Med 2013;368:2355-65

15. Demchuk AM, Hill MD, Barber PA, et al. Importance of early ischemic computed tomography changes using ASPECTS in NINDS rtPA stroke study. Stroke 2005;36:2110-15

16. Hill MD, Rowley HA, Adler F, et al. Selection of acute ischemic stroke patients for intra-arterial thrombolysis with pro-urokinase by using ASPECTS. Stroke 2003;34:1925-31

17. Harrison MJ, Marshall J. The variable clinical and CT findings after carotid occlusion: the role of collateral blood supply. J Neurol Neurosurg Psychiatry 1988;51:269-72

18. Bozzao L, Fantozzi LM, Bastianello S, et al. Early collateral blood supply and late parenchymal brain damage in patients with middle cerebral artery occlusion. Stroke 1989;20:735-40

19. Henderson RD, Eliasziw M, Fox AJ, et al. Angiographically defined collateral circulation and risk of stroke in patients with severe carotid artery stenosis: North American Symptomatic Carotid Endarterectomy Trial (NASCET) group. Stroke 2000;31:128-32

20. McVerry F, Liebeskind DS, Muir KW. Systematic review of methods for assessing leptomeningeal collateral flow. AJNR Am J Neuroradiol 2012;33:576-82

21. Smit EJ, Vonken EJ, van Seeters T, et al. Timing-invariant imaging of collateral vessels in acute ischemic stroke. Stroke 2013;44:2194-99

22. Menon BK, O’Brien B, Bivard A, et al. Assessment of leptomeningeal collaterals using dynamic $C T$ angiography in patients with acute ischemic stroke. J Cereb Blood Flow Metab 2013;33:365-71

23. Broderick JP, Palesch YY, Demchuk AM, et al. Endovascular therapy after intravenous t-PA versus t-PA alone for stroke. $N$ Engl J Med 2013;368:893-903

24. Kidwell CS, Jahan R, Alger JR, et al. Design and rationale of the mechanical retrieval and recanalization of stroke clots using embolectomy (MR RESCUE) trial. Int J Stroke 2014;9:110-16 